\title{
The Predictably Elusive Form II of Aspirin
}

Peddy Vishweshwar, ${ }^{\dagger}$ Jennifer A. McMahon, ${ }^{\dagger}$ Mark Oliveira, ${ }^{\ddagger}$ Matthew L.

Peterson ${ }^{\ddagger}$ and Michael J. Zaworotko ${ }^{*}, \dagger$

${ }^{\dagger}$ Department of Chemistry, University of South Florida, CHE205, 4202 E. Fowler Avenue, Tampa, Florida, 33620, USA

TransForm Pharmaceuticals, Inc., 29, Hartwell Avenue, Lexington, Massachusetts, 02421, USA.

\section{Supporting Information}

Appendix. Determination of salicylic acid content in bulk aspirin by HPLC.

Figure S1. Infrared spectra of Aspirin Form I (green) and Form II (red).

Figure S2. DSC of Aspirin Form I (green, $143.91^{\circ} \mathrm{C}$ ) and Form II (red, $135.5^{\circ} \mathrm{C}$ ).

Figure S3. Crystal packing of 1:1 co-crystal of aspirin and carbamazepine.

Table S4: Atom numbering and torsion angles for aspirin forms I and II.

Table S5. Hydrogen bonds for Aspirin Form II.

Table S6. Hydrogen bonds for 1:1 Aspirin and Carbamazepine co-crystal. 
Appendix. Determination of salicylic acid content in bulk aspirin.

The following work was performed to measure the salicylic acid content in samples of acetylsalicylic acid that were used to generate the form II polymorph of aspirin. HPLC was used to separate the salicylic and acetylsalicylic peaks and generate standard curves to measure their concentrations. It was determined that the presence of levetiracetam in solution did not affect the peaks measured by HPLC. Samples from Matt Peterson (MP-1 through MP-11) and Örn Almarsson (Orn-1 and Orn-11) were tested with the HPLC method. The Orn-11 sample had previously been determined to be form II.

The HPLC method used was:

Column: Waters Symmetry C18, $3.5 \mu \mathrm{m}, 4.6$ x 30mm

Isocratic $87.5 \% \mathrm{~A}, 12.5 \% \mathrm{~B}$

$\mathbf{A}=0.1 \%$ triflouroacetic acid in water

$\mathbf{B}=0.1 \%$ triflouroacetic acid in acetonitrile

Flow $=1.5 \mathrm{~mL} / \mathrm{min}$

Detector $=232 \mathrm{~nm}$

Table S1. Salicylic acid and aspirin concentrations from submitted HPLC samples. Sample concentrations were kept in a range were the standard curve error was less than $5 \%$.

\begin{tabular}{|c|c|c|c|c|c|c|c|c|c|}
\hline Sample & $\begin{array}{l}\text { HPLC } \\
\text { Name }\end{array}$ & $\begin{array}{c}\text { Area } \\
\left(\mu V^{*} s e c\right) \\
\text { Aspirin }\end{array}$ & $\begin{array}{c}\text { Area } \\
\left(\mu V^{*} \mathrm{sec}\right) \\
\text { Salicylic }\end{array}$ & $\begin{array}{l}\% \text { Amount } \\
\text { Aspirin }\end{array}$ & $\begin{array}{l}\% \text { Amount } \\
\text { Salicylic }\end{array}$ & $\begin{array}{c}\text { Amount } \\
\text { Aspirin } \\
(\mathrm{mg} / \mathrm{mL})\end{array}$ & $\begin{array}{l}\text { Amount } \\
\text { Salicylic } \\
\text { (mg/mL) }\end{array}$ & $\begin{array}{c}\text { Retention } \\
\text { Time } \\
\text { (min) } \\
\text { Aspirin }\end{array}$ & $\begin{array}{c}\text { Retention } \\
\text { Time } \\
\text { (min) } \\
\text { Salicylic }\end{array}$ \\
\hline MP-1 & MO-74-1 & 5731204 & 142713 & 98.04 & 1.96 & 0.35055 & 0.00697 & 3.281 & 5.199 \\
\hline MP-2 & MO-74-2 & 2787990 & 104888 & 97.16 & 2.84 & 0.17053 & 0.00512 & 3.297 & 5.202 \\
\hline MP-3 & MO-74-3 & 5180194 & 159209 & 97.54 & 2.46 & 0.31685 & 0.00778 & 3.282 & 5.203 \\
\hline MP-4 & MO-74-4 & 3498431 & 100640 & 97.72 & 2.28 & 0.21398 & 0.00492 & 3.303 & 5.219 \\
\hline MP-5 & MO-74-5 & 3556014 & 108841 & 97.76 & 2.24 & 0.2175 & 0.00532 & 3.308 & 5.22 \\
\hline MP-6 & MO-74-6 & 5000758 & 186883 & 97.14 & 2.86 & 0.30587 & 0.00913 & 3.3 & 5.22 \\
\hline MP-7 & MO-74-7 & 12268410 & 453186 & 97.15 & 2.85 & 0.7504 & 0.02213 & 3.275 & 5.214 \\
\hline MP-8 & MO-74-8 & 3746619 & 136711 & 97.03 & 2.97 & 0.22916 & 0.00668 & 3.3 & 5.214 \\
\hline MP-9 & MO-74-9 & 7886027 & 222362 & 97.77 & 2.23 & 0.48235 & 0.01086 & 3.279 & 5.204 \\
\hline MP-10 & MO-74-10 & 3982042 & 98793 & 97.99 & 2.01 & 0.24356 & 0.00483 & 3.304 & 5.218 \\
\hline MP-11 & MO-74-11 & 13408665 & 73152 & 99.51 & 0.49 & 0.82014 & 0.00357 & 3.27 & 5.216 \\
\hline USF Aspirin & MO-74-23 & 10717502 & 61473 & 99.54 & 0.46 & 0.65554 & 0.003 & 3.272 & 5.203 \\
\hline TPI Aspirin & MO-74-25 & 15938780 & 86428 & 99.59 & 0.41 & 0.9749 & 0.00422 & 3.248 & 5.192 \\
\hline Blank & Blank & 15564 & 2098 & 100 & 0 & 0.00095 & 0.0001 & 3.275 & 5.126 \\
\hline Orn-1(Form I) & MO-75-1 & 13060004 & 43772 & 99.75 & 0.25 & 0.79882 & 0.00214 & 3.263 & 5.199 \\
\hline $\begin{array}{c}\text { Orn -11 (Form } \\
\text { II) }\end{array}$ & MO-75-2 & 15535127 & 324944 & 98.34 & 1.66 & 0.95021 & 0.01587 & 3.245 & 5.191 \\
\hline
\end{tabular}


Table S2. Data from aspirin standards. Peak areas below 20,000 have very large errors.

\begin{tabular}{|r|l|l|l|l|l|l|r|r|}
\hline \multicolumn{2}{|c|}{$\begin{array}{c}\text { Standard Curve } \\
\text { Samples - Aspirin }\end{array}$} & \multicolumn{3}{c|}{ Aspirin } & $\begin{array}{c}\text { Predicted } \\
\text { Aspirin } \\
\text { concentration }\end{array}$ & \%Error \\
\hline & SampleName & & $\begin{array}{c}\text { Retention } \\
\text { Time } \\
(\mathrm{min})\end{array}$ & Amount & $\%$ Amount & $\begin{array}{c}\text { Area } \\
\left(\mu \mathrm{V}^{*} \mathrm{sec}\right)\end{array}$ & & \\
\hline 1 & Aspirin_10000X & 0.0001049 & 3.285 & 0.0001 & & 3549 & $2.17 \mathrm{E}-04$ & $\mathbf{1 1 7 . 0 8}$ \\
\hline 2 & Aspirin_1000X & 0.0010488 & 3.293 & 0.00105 & 100 & $\mathbf{2 1 8 7 8}$ & $1.34 \mathrm{E}-03$ & $\mathbf{2 7 . 4 4}$ \\
\hline 3 & Aspirin_100X & 0.010488 & 3.292 & 0.01049 & 100 & 188174 & $1.15 \mathrm{E}-02$ & 9.72 \\
\hline 4 & Aspirin_10X & 0.10488 & 3.286 & 0.10488 & 100 & 1822026 & $1.11 \mathrm{E}-01$ & 6.26 \\
\hline 5 & Aspirin_1X & 1.0488 & 3.241 & 1.0488 & 100 & 17136107 & $1.05 \mathrm{E}+00$ & -0.06 \\
\hline
\end{tabular}

Table S3. Data from salicylic acid standards. Peak areas below 20,000 have very large errors.

\begin{tabular}{|c|l|l|l|l|l|l|r|r|}
\hline \multicolumn{2}{|c|}{$\begin{array}{c}\text { Standard Curve } \\
\text { Samples - Salicylic }\end{array}$} & \multicolumn{3}{c|}{ Salicylic Acid } & $\begin{array}{c}\text { Predicted } \\
\text { salicylic } \\
\text { concentration }\end{array}$ & \%Error \\
\hline & SampleName & & $\begin{array}{c}\text { Retention } \\
\text { Time } \\
(\mathrm{min})\end{array}$ & Amount & $\%$ Amount & $\begin{array}{c}\text { Area } \\
\left(\mu \mathrm{V}^{*} \mathrm{sec}\right)\end{array}$ & & \\
\hline 6 & Salicylic_10000X & 0.0001078 & 5.152 & 0.00011 & & 4137 & $2.02 \mathrm{E}-04$ & $\mathbf{8 3 . 6 9}$ \\
\hline 7 & Salicylic_1000X & 0.001078 & 5.188 & 0.00108 & 100 & $\mathbf{2 3 5 1 3}$ & $1.15 \mathrm{E}-03$ & $\mathbf{6 . 3 4}$ \\
\hline 8 & Salicylic_100X & 0.01078 & 5.196 & 0.01078 & 100 & 226385 & $1.11 \mathrm{E}-02$ & 2.57 \\
\hline 9 & Salicylic_10X & 0.1078 & 5.185 & 0.1078 & 100 & 2287527 & $1.12 \mathrm{E}-01$ & 3.64 \\
\hline 10 & Salicylic_1X & 1.078 & 5.079 & 1.078 & 100 & 22062957 & $1.08 \mathrm{E}+00$ & -0.04 \\
\hline
\end{tabular}




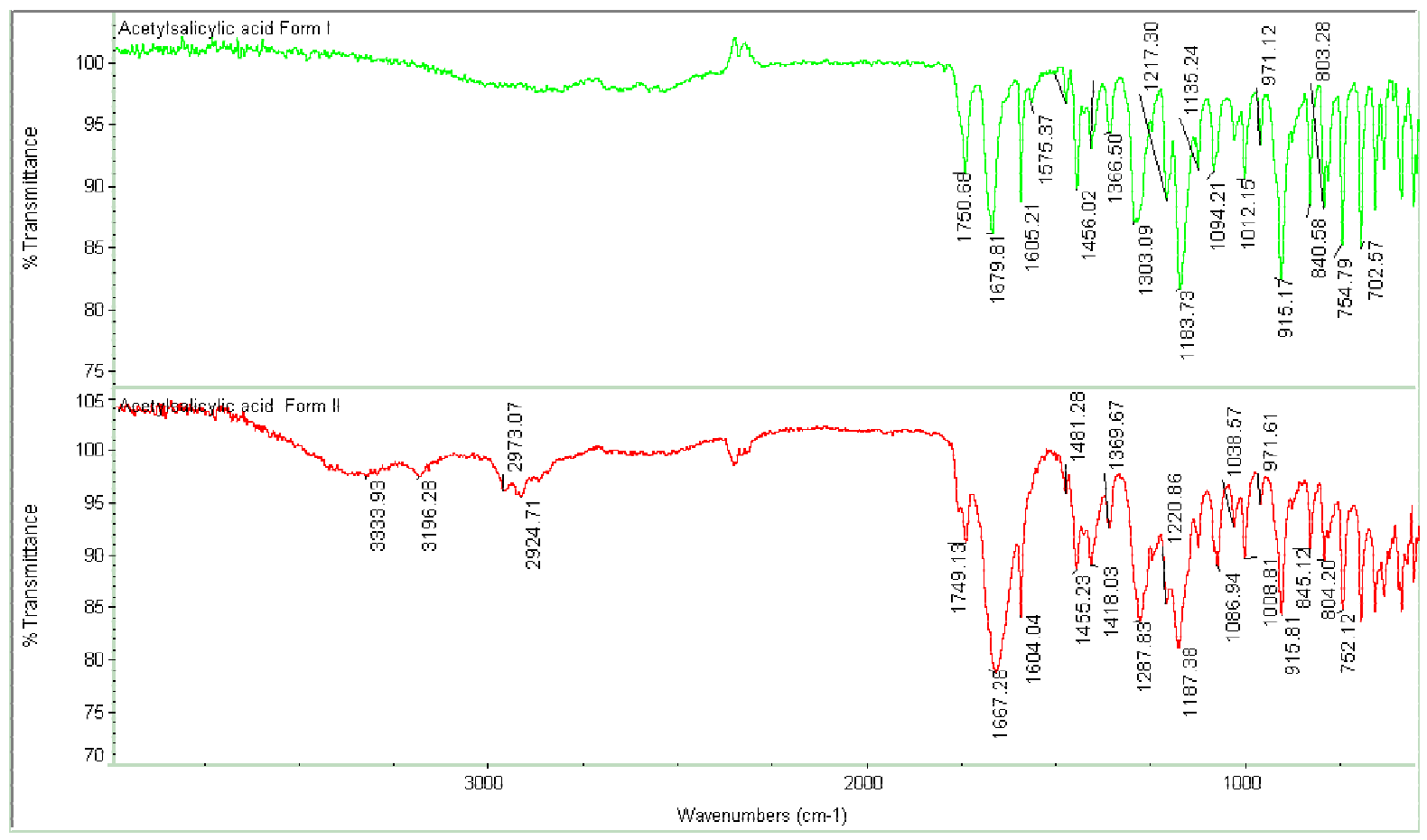

Figure S1. Infrared spectra of Aspirin Form I (green) and Form II (red).

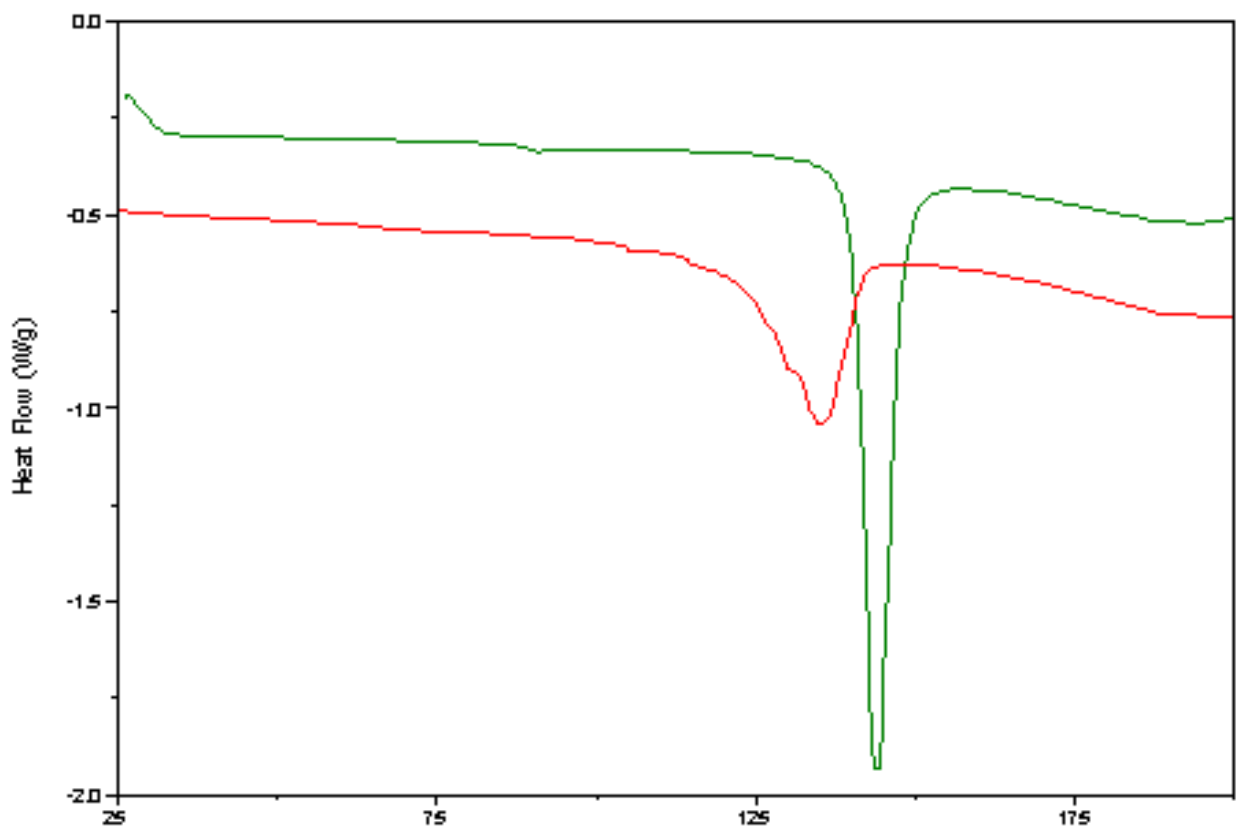

Figure S2. DSC of Aspirin Form I (green, $143.91{ }^{\circ} \mathrm{C}$ ) and Form II (red, $135.5^{\circ} \mathrm{C}$ ). 
Table S4. Atom numbering and torsion angles for aspirin forms I and II and S. L. Predicted form II.<smiles>CC(=O)Oc1ccccc1C(=O)O</smiles>

\begin{tabular}{||c|c|c|c||}
\hline & Form I & Form II & $\begin{array}{c}\text { S. L. Price predicted } \\
\text { form II (AK22) }\end{array}$ \\
\hline $\mathrm{C} 1-\mathrm{C} 2-\mathrm{O} 3-\mathrm{C} 8$ & $81.9(3)^{\mathbf{o}}$ & $84.0(18)^{\mathbf{o}}$ & $75.76^{\circ}$ \\
\hline $\mathrm{C} 2-\mathrm{O} 3-\mathrm{C} 8-\mathrm{O} 4$ & $3.6(4)^{\mathbf{o}}$ & $5.4(19)^{\mathbf{o}}$ & $4.36^{\mathbf{o}}$ \\
\hline $\mathrm{C} 2-\mathrm{C} 1-\mathrm{C} 7-\mathrm{O} 2$ & $1.2(2)^{\mathbf{o}}$ & $3.0(15)^{\mathbf{o}}$ & $5.99^{\mathbf{o}}$ \\
\hline
\end{tabular}
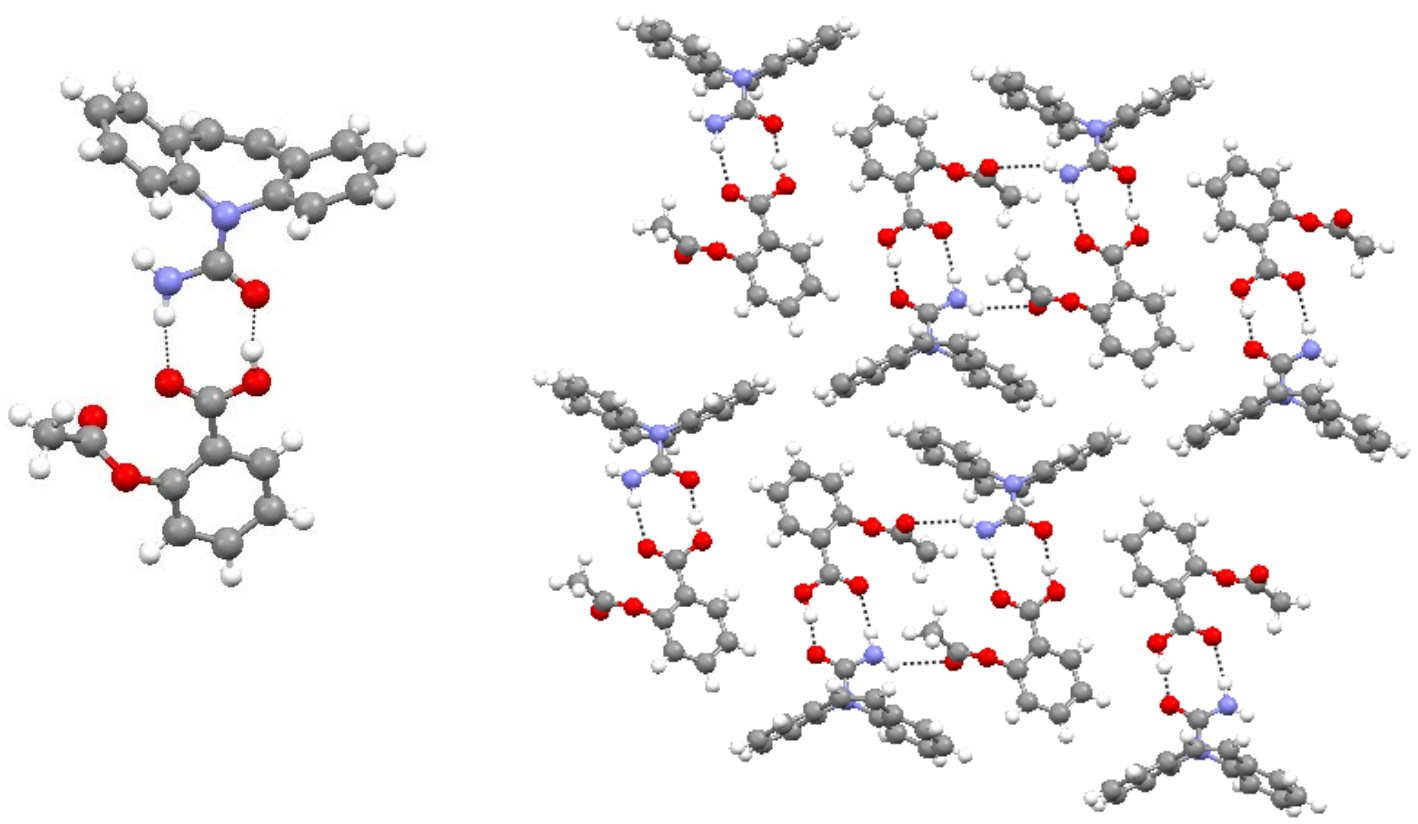

(a)

(b)

Figure S3. (a) A perspective view of the dimer that exists in the 1:1 co-crystal of aspirin and carbamazepine, which is sustained by the carboxylic acid-amide supramolecular heterosynthon. (b) Crystal packing of the dimers. 
Table S5. Hydrogen bonds for Aspirin Form II [ $\left[\AA\right.$ and $\left.{ }^{\circ}\right]$.

\begin{tabular}{lcccc}
\hline D-H...A & $\mathrm{d}(\mathrm{D}-\mathrm{H})$ & $\mathrm{d}(\mathrm{H} \ldots \mathrm{A})$ & $\mathrm{d}(\mathrm{D} \ldots \mathrm{A})$ & $<(\mathrm{DHA})$ \\
\hline $\mathrm{O}(1)-\mathrm{H}(1) \ldots \mathrm{O}(2) \# 1$ & 0.84 & 1.80 & $2.632(14)$ & 173.1 \\
$\mathrm{C}(9)-\mathrm{H}(9 \mathrm{~A}) \ldots \mathrm{O}(4)$ & 0.98 & 2.89 & $3.85(2)$ & 164.0 \\
\hline
\end{tabular}

Symmetry transformations used to generate equivalent atoms:

$\# 1-\mathrm{x}+1,-\mathrm{y},-\mathrm{z}+2$

Table S6. Hydrogen bonds for 1:1 Aspirin and Carbamazepine co-crystal [ $\left[\AA\right.$ and $\left.{ }^{\circ}\right]$.

\begin{tabular}{ccccc}
\hline D--H...A & $\mathrm{d}(\mathrm{D}-\mathrm{H})$ & $\mathrm{d}(\mathrm{H} \ldots \mathrm{A})$ & $\mathrm{d}(\mathrm{D} \ldots \mathrm{A})$ & $<(\mathrm{DHA})$ \\
\hline $\mathrm{O}(21)-\mathrm{H}(21 \mathrm{O}) \ldots \mathrm{O}(1) \# 1$ & 0.97 & 1.61 & $2.564(2)$ & 167.5 \\
$\mathrm{~N}(2)-\mathrm{H}(2 \mathrm{NA}) \ldots \mathrm{O}(22) \# 2$ & 0.95 & 1.98 & $2.914(3)$ & 168.4 \\
$\mathrm{~N}(2)-\mathrm{H}(2 \mathrm{NB}) \ldots \mathrm{O}(24) \# 3$ & 0.85 & 2.42 & $3.187(3)$ & 151.6 \\
\hline
\end{tabular}

Symmetry transformations used to generate equivalent atoms:

\#1 x,y,z+1 \#2 x,y,z-1 \#3 -x,-y+1,-z+1 